\title{
Gas Phase Sensing of Alcohols by Metal Organic Framework- Polymer Composite Materials
}

\author{
Sumit Sachdeva, ${ }^{\dagger}$ Sander J. H. Koper, ${ }^{\dagger}$ Anahid Sabetghadam, ${ }^{\dagger}$ Dimitri Soccol, ${ }^{\ddagger}$ Dirk J. Gravesteijn, ${ }^{\S}$ \\ Freek Kapteijn, ${ }^{\dagger}$ Ernst J. R. Sudhölter, ${ }^{\dagger}$ Jorge Gascon, $^{\dagger}$ and Louis C. P. M. de Smet ${ }^{*},,^{\dagger},{ }^{\circ}$ \\ ${ }^{\dagger}$ Delft University of Technology, Department of Chemical Engineering, Van der Maasweg 9, 2629 HZ Delft, Netherlands \\ ${ }^{\ddagger}$ NXP Semiconductors BV, Interleuvenlaan 80, 3001 Leuven, Belgium \\ ${ }^{\S}$ MESA+ Institute University of Twente, formerly NXP Semiconductors BV, Interleuvenlaan 80, 3001 Leuven, Belgium \\ "Wageningen University \& Research, Laboratory of Organic Chemistry, Stippeneng 4, 6708 WE, Wageningen, Netherlands
}

\author{
Supporting Information
}

\begin{abstract}
Affinity layers play a crucial role in chemical sensors for the selective and sensitive detection of analytes. Here, we report the use of composite affinity layers containing Metal Organic Frameworks (MOFs) in a polymeric matrix for sensing purposes. Nanoparticles of $\mathrm{NH}_{2}-\mathrm{MIL}-53(\mathrm{Al})$ were dispersed in a Matrimid polymer matrix with different weight ratios (0-100 wt \%) and drop-casted on planar capacitive transducer devices. These coated devices were electrically analyzed using impedance spectroscopy and investigated for
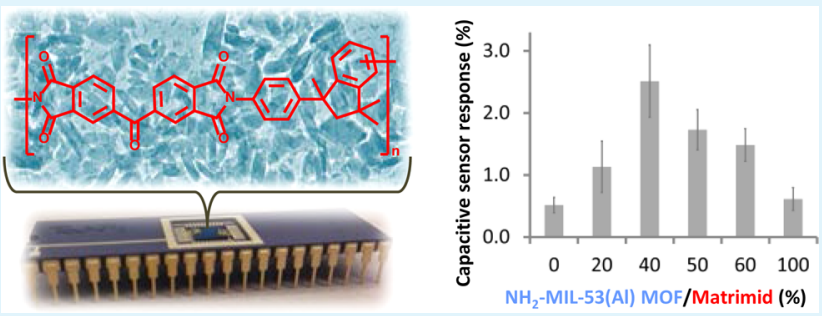
their sensing properties toward the detection of a series of alcohols and water in the gas phase. The measurements indicated a reversible and reproducible response in all devices. Sensor devices containing 40 wt \% $\mathrm{NH}_{2}-\mathrm{MIL}-53$ (Al) in Matrimid showed a maximum response for methanol and water. The sensor response time slowed down with increasing MOF concentration until 40 wt $\%$. The half time of saturation response $\left(\tau_{0.5}\right)$ increased by $\sim 1.75$ times for the 40 wt \% composition compared to devices coated with Matrimid only. This is attributed to polymer rigidification near the MOF/polymer interface. Higher MOF loadings $(\geq 50 \mathrm{wt} \%)$ resulted in brittle coatings with a response similar to the $100 \mathrm{wt} \%$ MOF coating. Cross-sensitivity studies showed the ability to kinetically distinguish between the different alcohols with a faster response for methanol and water compared to ethanol and 2-propanol. The observed higher affinity of the pure Matrimid polymer toward methanol compared to water allows also for a higher uptake of methanol in the composite matrices. Also, as indicated by the sensing studies with a mixture of water and methanol, the methanol uptake is independent of the presence of water up to $6000 \mathrm{ppm}$ of water. $\mathrm{The}_{\mathrm{NH}}-\mathrm{MIL}-53(\mathrm{Al})$ MOFs dispersed in the Matrimid matrix show a sensitive and reversible capacitive response, even in the presence of water. By tuning the precise compositions, the affinity kinetics and overall affinity can be tuned, showing the promise of this type of chemical sensors.
\end{abstract}

KEYWORDS: metal organic frameworks, composites, mixed matrix membranes, impedance spectroscopy, capacitive detection, gas sensors

\section{INTRODUCTION}

Detection of analytes in the gas phase is important in environmental, security, health, food, and industrial processes requiring detection and quantification in a fast, accurate, and reversible manner. ${ }^{1-3}$ In recent years, studies have focused on developing chemical coatings as a selective affinity layer in sensors using nanomaterials ${ }^{1,4,5}$ and polymeric films. ${ }^{6,7}$ The uptake of analytes in the affinity layer leads to changes in the physical properties of the films which can be monitored by the transducer beneath. Often, metal oxides are used as the affinity layer which usually require high operation temperatures $(>200$ ${ }^{\circ} \mathrm{C}$ ) and do show a significant cross-sensitivity in most cases. ${ }^{8,9}$

Metal organic frameworks (MOFs) are a new emerging class of materials of which their specific affinity properties can be attractive for use as selective substrates in chemical gas phase sensors operating at low temperature. ${ }^{2,10,11}$ These materials consist of metal ions linked by organic ligand molecules forming porous, crystalline frameworks. ${ }^{11,12}$ Their high porosity and tunability of their structure and selectivity make them promising for applications not only for sensing, but also for gas separation, storage, and catalysis. ${ }^{1-14}$

To date, sensor studies using MOFs have focused mainly on changing their luminescence properties. ${ }^{15,16}$ Recent advances in the preparation of thin films of MOFs have opened their potential in the sensor industry beyond luminescence-based sensors. ${ }^{13,17}$ These recent studies are mainly based on techniques like Quartz-crystal Microbalance (QCM), Surface Acoustic Waves (SAW), and cantilevers, which utilize changes

Received: February 22, 2017

Accepted: April 11, 2017

Published: April 25, 2017 


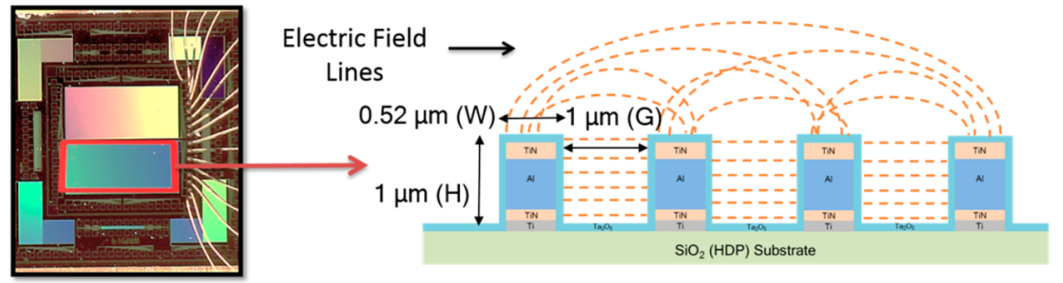

(a)

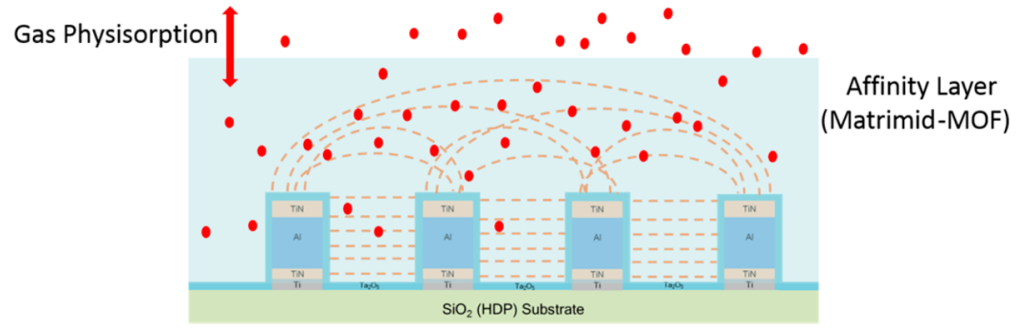

(b)

Figure 1. (a) The sensor device consisting of meander-patterned electrodes with height $(H)$, width $(W)$ and gap $(G)$ of $1 \mu \mathrm{m}, 0.52 \mu \mathrm{m}$ and $1 \mu \mathrm{m}$, respectively. (b) Schematic representation of the device with the higher dielectric affinity layer and gas physisorption within this layer.

in mass, mechanical properties for analyte detection in MOFbased sensor devices. ${ }^{17}$ Also, studies utilizing changes in the electrical properties of MOFs have shown promising application of MOFs as selective affinity layers for sensors. ${ }^{18-20}$ Specifically, MOF-based capacitive sensors offer advantages over resistive sensors due to the insulating nature of MOFs. Compared to, e.g., field-effect transistors, capacitive sensors can be fabricated relatively easily at low cost on a large variety of substrates. Furthermore, they have a low energy consumption and allow different electrode architectures to reduce noise and improve sensitivity.

Additionally, the use of polymeric composite materials with MOFs can combine the properties of both polymers and MOFs for obtaining superior sensing performance. This has also been exploited in gas separation applications. ${ }^{21,22}$ The enhanced compatibility of polymers and MOFs contribute also to their promising role in producing new sensor selector layers. ${ }^{21-23}$ Studies with polymeric sensors containing filler molecules such as metal oxides, carbon nanotubes, and graphene have already shown improvement of the intrinsic sensing properties of polymers. $^{24-26}$ Moreover, the use of composite materials can allow for easy and cost-effective integration with semiconductor technologies utilizing existing principles for synthesizing polymeric coatings. ${ }^{27,28}$

Recently, we reported a simple methodology for developing polymer-MOF composite films using Matrimid 5218 and $\mathrm{NH}_{2}-$ MIL-53(Al) for sensing applications. ${ }^{29}$ Matrimid 5218 belongs to the class of fully imidized polyimides with a high thermal and mechanical stability ${ }^{30,31}$ and $\mathrm{NH}_{2}-\mathrm{MIL}-53(\mathrm{Al})$ is one of the most well-studied MOFs with aluminum as the metal ion and amino-terephthalic acid as the organic ligand. ${ }^{31,32}$ It was observed that incorporation of these MOF particles inside the Matrimid polymer matrix enhances the sensitivity of the sensor devices. Matrimid and $\mathrm{NH}_{2}$-MIL-53(Al) have also shown significant compatibility during membrane separation studies leading to superior gas separation performances. ${ }^{31}$ The presence of these MOF particles inside the polymeric matrix alter their physical and chemical properties. ${ }^{29,31}$ For example, it has been observed from membrane studies that variations of the concentration of MOF particles can lead to alterations in the gas transport and adsorption properties. ${ }^{31,33}$ Furthermore, upon blending the overall dielectric constant-and hence the electrical polarizability-changes. ${ }^{34}$ These differences in the properties with varying MOF concentration can be quite significant for a pair of MOF and polymer.

In this study, we focused on developing planar transducer sensor devices by the deposition of composite films consisting of different amounts of $\mathrm{NH}_{2}$-MIL-53(Al) in Matrimid. These devices are meander-patterned planar aluminum electrodes having a width $(W)$ of $0.52 \mu \mathrm{m}$, a gap $(G)$ of $1 \mu \mathrm{m}$, and an electrode area of $\sim 2.1 \mathrm{~mm}^{2}$ (Figure 1). About $95 \%$ of the electrical field lines stay within the distance equal to the sum of $W$ and $G$ in a perpendicular orientation. ${ }^{35-37}$ Our bare electrodes show a capacitance of $\sim 22 \mathrm{pF}$. After deposition of polymer-MOF affinity layers of $10 \mu \mathrm{m}$ in thickness onto our electrode, the capacitance increases to $28-36 \mathrm{pF}$ as a result of the higher dielectric constant of the deposited layer $\left(\varepsilon_{\mathrm{r}}=\right.$ $\sim 3.5-4.5)$ as compared to air $\left(\varepsilon_{\mathrm{r}}=1\right)$, as previously discussed. ${ }^{29}$ If analyte molecules are adsorbed in the polymerMOF matrix, then the overall dielectric constant will also change, resulting in a capacitance change. ${ }^{29}$ We have performed such sensing experiments using methanol and water as the analyte, varying the polymer-MOF composition ratio. In addition, cross-sensitivity studies with ethanol and 2-propanol and temperature-dependent response studies were performed.

\section{EXPERIMENTAL SECTION}

2.1. Materials. The polyimide (PI) Matrimid 5218 was obtained from Huntsman Advanced Materials and was used as the polymeric matrix. Methanol, ethanol, and 2-propanol were obtained from SigmaAldrich at highest purity. $\mathrm{N}_{2}$ gas (99.99\%) was obtained from Linde Gas and used as carrier gas in our sensor experiments. All other chemicals were obtained from Sigma-Aldrich and used as received.

2.2. Device Fabrication. The fabrication of the devices was performed as described previously. ${ }^{29}$ Briefly, the active electrode area consisted of aluminum metal stacked between TiN layers over underlying dielectric layer of $\mathrm{SiO}_{2}$ to mimic the final layer of a typical $140 \mathrm{~nm}$ CMOS platform. Devices were processed to obtain meanderpatterned planar aluminum electrodes by photolithography. These 


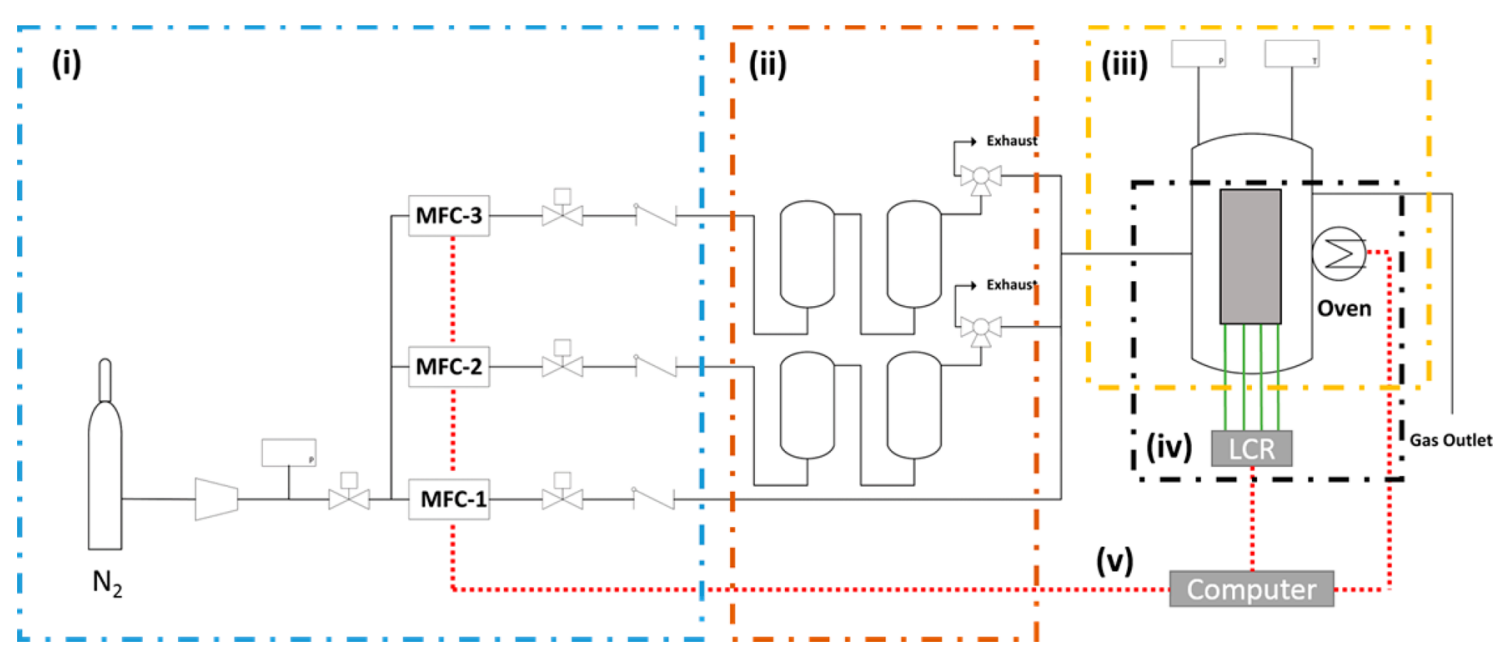

Figure 2. Schematic of the measurement setup consisting of a (i) gas mixing unit with mass-flow controllers, (ii) vapor generation system with series of bubblers, (iii) temperature-controlled measurement chamber, (iv) electrical measurement unit, and (v) instrument control and data logging using LabVIEW and computer.

electrodes consist of 4 parallel electrodes with a meander pattern with line width $(W)$ of $0.52 \mu \mathrm{m}$, a gap $(G)$ of $1 \mu \mathrm{m}$ and a total electrode area of $\sim 2.1 \mathrm{~mm}^{2}$. First, $\mathrm{Si}_{3} \mathrm{~N}_{4}$ was deposited on the backside of the $\mathrm{p}$ doped $\mathrm{Si}$ wafer to avoid contamination of the Si during the wet cleaning steps. A high-density plasma CVD silicon dioxide (with a thickness of $3 \mu \mathrm{m}$ ) was deposited to represent the intermetal dielectric, followed by the deposition of the metal stack. This metal stack consists of a $25 / 25 \mathrm{~nm} \mathrm{Ti} / \mathrm{TiN}$ adhesion bilayer at the bottom and followed by a $800 \mathrm{~nm}$ thick layer of aluminum and a $50 \mathrm{~nm}$ layer of $\mathrm{TiN}$ at the top. $\mathrm{The} \mathrm{Ti} / \mathrm{TiN}$ and $\mathrm{TiN}$ layers were introduced to improve the adhesion, to decrease reflectivity, and also to function as a stopping layer during the etching processes. Afterward, the $\mathrm{Al}$ metal layer for the device was patterned by lithography targeting the required width and spacing dimensions. After photoresist patterning, the $\mathrm{Al}$ lines were dry etched to obtain 4 parallel meander-patterned aluminum lines. Subsequently, a $\mathrm{Ta}_{2} \mathrm{O}_{5}$ line of $40 \mathrm{~nm}$ was deposited by CVD to serve as a moisture diffusion barrier.

2.3. Synthesis of the MOF Nanoparticles. $\mathrm{NH}_{2}-\mathrm{MIL}-53(\mathrm{Al})$ nanoparticles were synthesized by hydrothermal treatment using 2aminoterephthalic acid as organic ligand and aluminum nitrate using the procedure previously described. ${ }^{22,38}$ Therefore, $1.902 \mathrm{~g}(=10.5$ mmol) 2-aminoterephthalic acid was dissolved in $10.5 \mathrm{~mL} 2 \mathrm{M}$ aqueous $\mathrm{NaOH}$ solution after which the volume was increased to 75 $\mathrm{mL}$ using distilled water. $3.935 \mathrm{~g} \mathrm{Al}\left(\mathrm{NO}_{3}\right)_{3} .9 \mathrm{H}_{2} \mathrm{O}(=10.5 \mathrm{mmol})$ was dissolved in $75 \mathrm{~mL}$ distilled water in a separate tube. Both solutions were mixed and stirred under reflux conditions (393 K) for 3 days. The formed MOF particles were filtered under vacuum and washed with $96 \%$ ethanol twice. The material was then washed in DMF $(\sim 100$ $\mathrm{mL})$ and methanol $(\sim 100 \mathrm{~mL})$ consecutively at 403 and $363 \mathrm{~K}$ overnight, respectively to efficiently remove any unreacted ligand. Finally, the powder was filtered and washed with ethanol twice and dried at $373 \mathrm{~K}$ in vacuum overnight.

2.4. Preparation of the Device Affinity Layers. The polyimide (PI) Matrimid 5218 was pretreated at $453 \mathrm{~K}$ overnight under vacuum to remove any physically adsorbed water. Different loadings $(0,20,40$, 50 , and $60 \mathrm{wt} \%$ ) of $\mathrm{NH}_{2}$-MIL-53(Al) in Matrimid were prepared by dissolving the calculated amount of materials in tetrahydrofuran (THF) in such a way that the total weight of both components was 6 wt $\%$ ( 5.5 wt $\%$ for 50 and 60 wt \% loadings). First, the required amount of MOF particles for each concentration was dispersed in 940 $\mathrm{mg}$ of THF by ultrasonication for $15 \mathrm{~min}$. Then, the remaining amount of polymer (for each desired concentration) was added to this dispersion and stirred overnight. In the case of MOF-free polyimide coatings, $60 \mathrm{mg}$ of PI was dissolved in $940 \mathrm{mg}$ of THF and stirred overnight. For the preparation of coatings containing pure MOF (100 wt \%, i.e., without PI), $22.5 \mathrm{mg}$ of MOF nanoparticles were dispersed in $480 \mathrm{mg}$ of THF. Before the deposition of the solutions over devices and reference silicon substrates, the solutions were subjected to three alternative cycles of ultrasonication and mixing of 15 min each to ensure good dispersion of MOF particles. ${ }^{22,31}$ The dispersed solution was then cast over the reference substrate and transducer devices with $5 \mu \mathrm{L}$ drops under a solvent-saturated environment to reduce evaporation of the THF solvent. This ensures that the formed coatings do not crack and remain uniform. Once dried, the coated devices were treated at $453 \mathrm{~K}$ in vacuum for $4 \mathrm{~h}$.

2.5. Materials Characterization. Scanning Electron Microscopy (SEM) images of the composite films were acquired using a JEOL JSM 6010LA microscope after sputtering the sample with a conductive gold layer. TEM images of MOF particles were acquired with a JEOL JEM1400 instrument. XRD patterns were recorded with a Bruker D8 Advance diffractometer with Co- $K \alpha$ radiation $(\lambda=1.78897 \AA)$. The diffraction pattern was scanned with a step size of $0.02^{\circ}$ and a scan speed of $0.2 \mathrm{~s}$ per step. A DEKTAK 8 profilometer was used to determine the thickness of the composite films over reference silicon substrates with a force of $0.3 \mathrm{mg}$ and a horizontal and vertical resolution of $0.5 \mu \mathrm{m}$ and $4 \mathrm{~nm}$, respectively. For $40-60 \mathrm{wt} \% \mathrm{NH}_{2}$ MIL-53(Al)/Matrimid devices, the thickness was measured with a Nikon MUC-151 micrometer because of the brittleness of the composite layer. Impedance measurements of the sensor devices were carried out with an HP 4284A LCR meter in a four-probe configuration.

2.6. Gas Sensing Measurements. An automated gas mixing and detection setup has been designed and built for monitoring the behavior of gas sensor devices using impedance measurements on exposure to analytes and vapors in the gas phase. ${ }^{29}$ Figure 2 provides a schematic lay-out. The major part of the equipment consists of a (i) gas mixing unit containing mass flow controllers for providing desired flow rates of the gases, (ii) vapor generation system with a series of bubblers for analysis of individual and mixtures of vapors, (iii) temperature-controlled measurement chamber to analyze the components in the gas phase using sensor devices under test, (iv) electrical measurement units for monitoring perturbations in the measurement chamber, and (vi) instrument controller and data logger system for controlling mass flow controllers, electrical instruments and recording data using LabVIEW-based software.

The gas mixing units were connected with the calibrated gas bottles to provide gases with desired concentration and flow rates to the measurement chamber. The addition of vapors was achieved by passing the inert gas $\left(\mathrm{N}_{2}\right)$ at atmospheric pressure through a series of bubblers to generate a saturated stream of $\mathrm{N}_{2}$ with the vapor at room temperature. The vapor concentration was calculated with the Antoine Equation: ${ }^{39,40}$ 

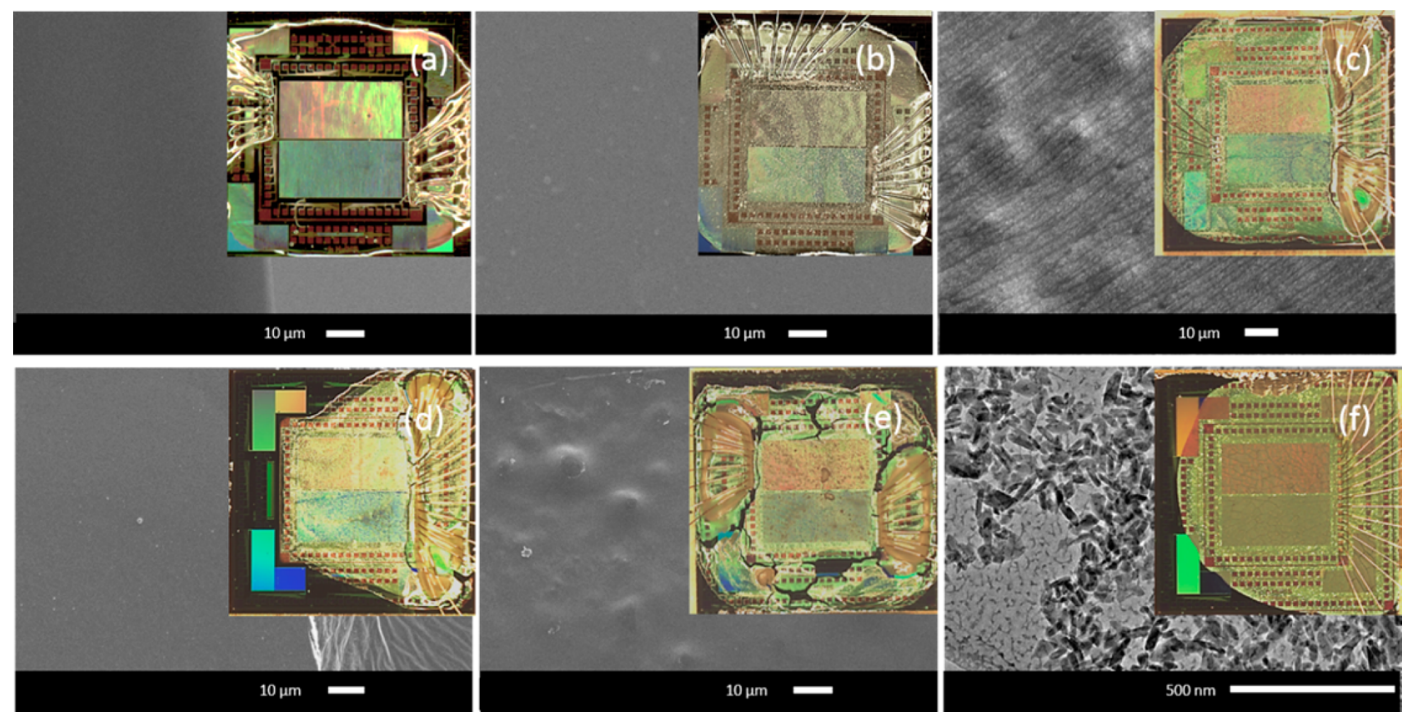

Figure 3. SEM micrographs and optical images (inserts) of the casted layers of $\mathrm{NH}_{2}$-MIL-53(Al)-Matrimid composites with different $\mathrm{MOF}$ concentrations: (a) 0, (b) 20, (c) 40, (d) 50, and (e) 60 wt \%. (f) TEM micrograph of $\mathrm{NH}_{2}$-MIL-53(Al) nanoparticles along with the optical image of a casted device with only MOF particles (100 wt \%).
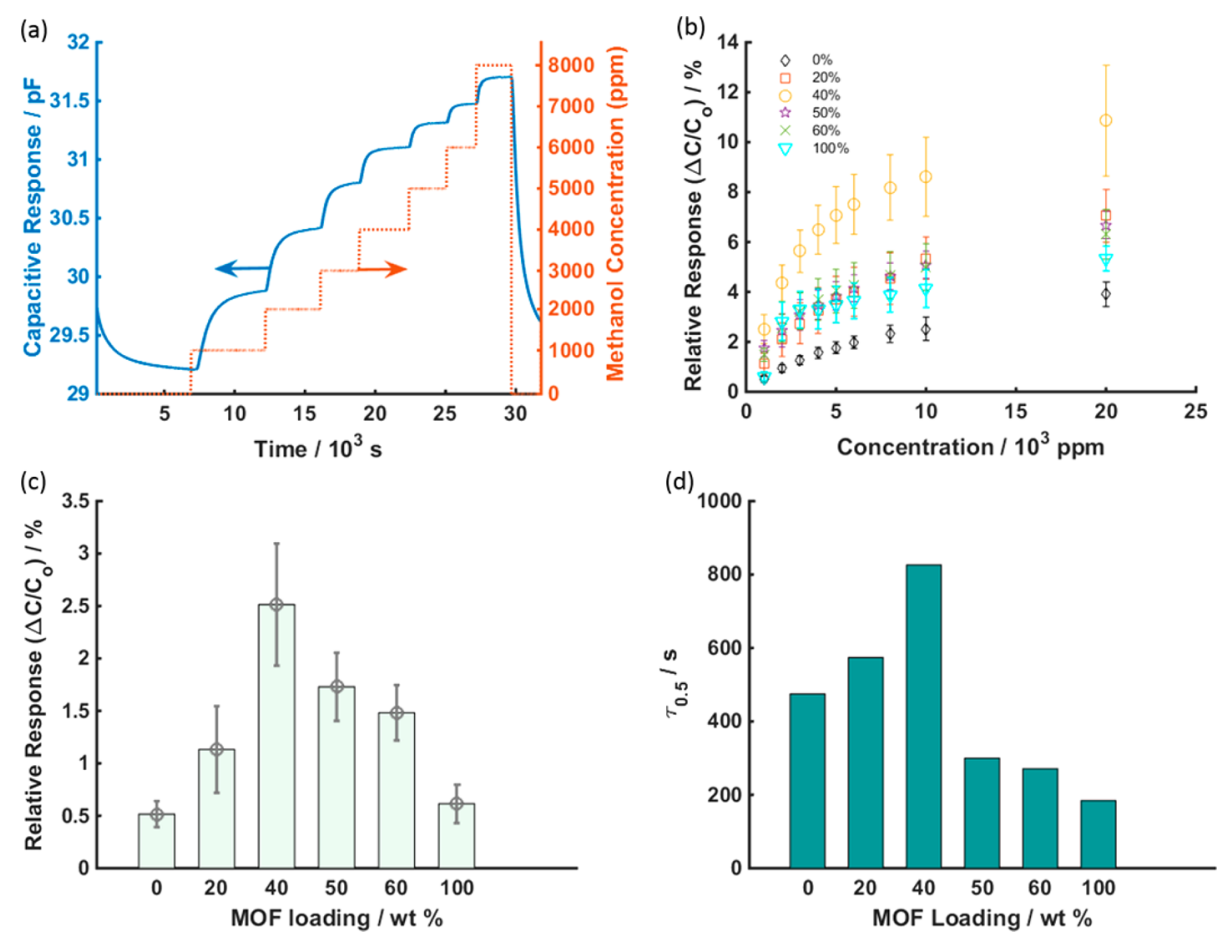

Figure 4. (a) Quantitative, cumulative response toward methanol by a sensor device coated with a thin layer of $40 \mathrm{wt} \% \mathrm{NH}-\mathrm{MIL}-53(\mathrm{Al})$ nanoparticles in Matrimid. (b) Comparison of the sensor response by devices with different ratios of $\mathrm{NH}_{2}-\mathrm{MIL}-53$ (Al) and Matrimid to the exposure of different concentrations of methanol. (c) Comparison of the response of these sensor devices toward $1000 \mathrm{ppm}(0.1 \%)$ of methanol. (d) The halftime for response saturation $\left(\tau_{0.5}\right)$ for these sensor devices on the exposure to $20000 \mathrm{ppm}(2 \%)$ of methanol. The error-bars were obtained from the measurements done on three independently replicated devices.

$$
\log P^{\circ}=A-\frac{B}{T+C}
$$

where $P^{o}$ is the saturated vapor pressure of the analyte, and $T$ is the temperature of the bubbler. $A, B$, and $C$ are analyte-dependent constants.

Sensing measurements were carried out using an HP 4284A precision LCR meter at a frequency of $20 \mathrm{kHz}$ and a voltage of $1 \mathrm{~V}$ in a four-probe arrangement. The devices were kept at a controlled temperature of $28{ }^{\circ} \mathrm{C}$ and a total flow of $200 \mathrm{~mL} / \mathrm{min}$. During the measurement, the desired concentration of an analyte component in dry $\mathrm{N}_{2}$ was passed through the measurement chamber having a volume of $\sim 400 \mathrm{~mL}$. After stabilization and equilibration, the sensors were recovered to the baseline by replacing the stream of $\mathrm{N}_{2}$ with the analyte by dry $\mathrm{N}_{2}$ only.

The frequency analyzer and the gas mixing setup were automated using a custom-built LabVIEW program to control the parameters such as flow rates, voltage, temperature, and carry out the time-based and frequency-based measurements. 


\section{RESULTS AND DISCUSSION}

3.1. Sensing Layer Formation. The crystallinity of the synthesized $\mathrm{NH}_{2}$-MIL-53(Al) MOFs nanoparticles was confirmed with powder X-ray Diffraction (XRD) ${ }^{31}$ (Figure S1 of the Supporting Information). The structure of the MOF consists of $\mathrm{AlO}_{4}(\mathrm{OH})_{2}$ octahedra coordinated to 2-aminoterephthalic acid and mainly exhibited a narrow-pore (np) configuration. ${ }^{31}$ The nanoparticles had a rod-shaped morphology and dimensions of $\sim 56 \times \sim 17 \mathrm{~nm}^{2}$ (Figures 3f and S2).

In order to investigate the role of the MOF particle concentration in the affinity layer for sensing, sensor devices were prepared with different filler ratios (0-60 wt \%) of $\mathrm{NH}_{2}$ MIL-53(Al) in a matrix of Matrimid. The polymer-MOF mixture in tetrahydrofuran (THF) was drop-casted in a solventsaturated environment onto the sensor devices and reference silicon substrates to obtain a $10 \mu \mathrm{m}$ thick layer (Figure S3). The thickness was kept at $10 \mu \mathrm{m}$ to ensure that the electrical field lines of the transducer stay inside the polymeric layer. ${ }^{29}$ This is three times higher than the spatial wavelength $(\sim 3 \mu \mathrm{m})$ of the transducer and ensures that deposition variations do not influence the equilibrium sensor response. The coated region was kept larger than the central active region (Figures 1 and 3) to maintain uniformity in the active region. The differences in the deposited composite layers can be seen with SEM (Figures 3a,b) and optically (Figure 3, insets). The surface coating turned slightly opaque due to the presence of agglomerated MOF particles. The roughness of the material was increased. At further increased MOF loadings, opacity also increased and the layer became brittle. At loadings above $50 \mathrm{wt} \%$ the layer showed cracks (Figures 3d,e and S4). For comparison, MOF particles ( $100 \mathrm{wt} \%$, i.e, in the absence of polymer matrix) were also deposited over the sensor devices (Figure 3f).

3.2. Quantitative Investigation of the Sensing Performance. Sensor devices coated with layers containing different MOF loadings were exposed to increasing concentrations of methanol in $\mathrm{N}_{2}$ at $28{ }^{\circ} \mathrm{C}$. Methanol was selected as the primary analyte due to the good sensitivity of $\mathrm{NH}_{2}$-MIL53(Al) and Matrimid for this alcohol. ${ }^{29}$ The capacitive changes on the exposure to methanol were monitored by using impedance spectroscopy with a frequency of $20 \mathrm{kHz}$ and a voltage of $1 \mathrm{~V}$. The sensor devices were first exposed to dry $\mathrm{N}_{2}$ in the measurement chamber until a stable baseline was achieved. Next, methanol vapors were introduced at different concentrations using a series of two bubblers (see Section 2.6 for details). Figure $4 \mathrm{a}$ shows a typical response of a sensor device covered with a PI coating containing $40 \mathrm{wt} \%$ of MOF nanoparticles toward different concentrations of methanol at 28 ${ }^{\circ} \mathrm{C}$. The methanol concentration was kept between 1000 and $20000 \mathrm{ppm}(0.1-2 \%)$, which is in line with industrial requirements. ${ }^{41,42}$ Lower concentrations (below $100 \mathrm{ppm}$ ) were not reliable due to the equipment limitations.

The capacitive response of the sensor device increases on exposure to methanol in the measurement chamber $(t=\sim 7000$ $s$ in Figure $4 a)$. The capacitive response further increased upon increasing methanol concentrations. Furthermore, the response was completely reversible as the initial baseline was recovered on replacing the methanol containing $\mathrm{N}_{2}$ gas by dry $\mathrm{N}_{2}$ (Figures 4a and S5).

The sensitivity of our devices with different amounts of MOF in the polymer matrix toward methanol is plotted in Figure $4 \mathrm{~b}$. The response was normalized by $C_{0}$, the capacitance in dry $\mathrm{N}_{2}$, yielding a relative change in capacitance $\left(\Delta C / C_{0}\right)$ allowing easy and accurate comparison of the different sensor devices. The inclusion of these porous nanoparticles inside the polymer matrix enhances the overall signal response, e.g., the addition of $20 \mathrm{wt} \%$ of MOF more than doubles the sensor response to methanol. This increase in the sensitivity is due to the additional adsorption sites introduced by the $\mathrm{NH}_{2}-\mathrm{MIL}-53(\mathrm{Al})$ along with the intrinsic adsorption capacity of the Matrimid. ${ }^{29,38,43}$ The response increased further upon increasing amounts of MOF in the polymeric layer, resulting in a 5 -fold increase (over exposure to $1000 \mathrm{ppm}$ methanol) for the $40 \mathrm{wt}$ $\%$ MOF loading as compared to the MOF-free Matrimid layer (Figure $4 b, c)$. Further, the maximum capacitive response was observed at $40 \mathrm{wt} \%$ (Figure $4 \mathrm{c})$. Such a response ( $\sim 750 \mathrm{fF}$ of capacitance change) is clearly higher than the detection limit of the instrument $(0.1 \mathrm{fF})$, making the current approach suitable for measuring methanol within most industrial relevant concentrations $(0-3000 \mathrm{ppm})$ at ambient conditions. ${ }^{41}$ It also allows to compete well with the commercially available methanol sensors (having a measurement range within 0$10000 \mathrm{ppm})$. The observed decrease in the response at a higher wt \% indicates a transition from a polymer-limited adsorption to a MOF-limited adsorption (Figure 4b,c).

We attribute this reduction in response to the decreased adhesion between the composite layers and transducer at loadings above 40 wt \%. Formation of brittle and cracked films at these loadings, as observed by SEM, supports this line of reasoning (Figure 3). Additionally, this decreased signal transduction behavior is also clearly visible in the sensor response of $100 \mathrm{wt} \% \mathrm{MOF}$ devices as the high adsorption capacity of $\mathrm{NH}_{2}-\mathrm{MIL}-53(\mathrm{Al})$ is not completely reflected in the sensor response (Figures $4 \mathrm{~b}$ and S6). Moreover, percolation of methanol molecules from MOF particle to MOF particle at such high loadings (above $40 \mathrm{wt} \%$ ) ensures that the adsorption process is directly determined by the MOF. ${ }^{23}$ The slightly higher response in these devices compared to that for the 100 wt \% MOF-coated device (Figure 4b,c) can be explained by additional adsorption in the Matrimid phase, which covers these nanoparticles partially.

The response kinetics provide further insights into the adsorption process. Interestingly, considering the time taken to reach half the saturation response $\left(\tau_{0.5}\right)$, the $\tau_{0.5}$ is observed to increase (slower process) with increasing the amount of $\mathrm{NH}_{2}$ MIL-53( $\mathrm{Al})$ in the Matrimid matrix for the lower concentration regime (0-40 wt \%) (Figure $4 \mathrm{~d}$ ). The observed increase of $\tau_{0.5}$ is attributed to rigidification of the polymer matrix near the polymer-MOF interface. ${ }^{44,45}$ The solvent evaporation can induce stress in the polymeric matrix around the filler molecules and reduce the relaxation of the polymeric chains close to the MOF surface while drying takes place. This would lead to a decrease in the free volume close to the surface and hence reduction in the permeability. This has also been observed before in membranes prepared by spin-coating layers of a glassy polymer (PSF-Udel) with $\mathrm{NH}_{2}$-MIL-53(Al). ${ }^{45}$ In addition, partial penetration of the Matrimid polymer chains into the micropores of $\mathrm{NH}_{2}-\mathrm{MIL}-53(\mathrm{Al})$ can also affect the transport of methanol to the MOF particles. ${ }^{45}$ Also, as shown in Figure $4 \mathrm{~d}, \tau_{0.5}$ drops by a factor of approximately 3 (faster process) at a MOF loading of $\geq 50$ wt $\%$ when compared with devices containing $40 \mathrm{wt} \%$ of MOF. At loadings of 50 and 60 wt $\%$ the response times also became significantly closer to the one of the MOF-coated device (100 wt \%). This observation confirms the direct accessibility of the MOF particles to the exposed environment due to cracks and to a thin or partial 
coverage of $\mathrm{NH}_{2}$-MIL-53(Al) particles with the (rigidified) Matrimid.

Moreover, on the assumption that the sensor response is proportional to the adsorbed concentration of the analyte in the composite matrix, ${ }^{29}$ the nonlinear response of different devices with varying methanol concentrations (Figure $4 \mathrm{~b}$ ) was fitted to the Langmuir adsorption model as shown in eq 2:

$$
\nu=\nu_{\max } \times \frac{K_{\mathrm{eq}} \times p_{\mathrm{MeOH}}}{1+K_{\mathrm{eq}} \times p_{\mathrm{MeOH}}}
$$

where $v, v_{\max }, K_{\text {eq }}$, and $p_{\mathrm{MeOH}}$ indicate the observed response $\left(\Delta C / C_{0}\right)$, maximal (saturation) response $\left(\Delta C_{\max } / C_{0}\right)$, adsorption equilibrium constant, and partial pressure of the analyte, respectively. $v$ and $v_{\max }$ are represented as normalized response (\%) for accurate comparison between different devices as the $C_{0}$ varies in each device due to the differences in the dielectric properties of composite films. Since the concentration range was still in the lower regime of the adsorption isotherm, we assumed that the adsorption behavior is still following the Langmuir model. The fitting was carried out on the average response by devices with different MOF loadings in the polymer matrix (Figure S7). The obtained parameters are listed in the Table 1, showing that there is good agreement with the experimental data in all the polymer-MOF coatings $\left(R^{2} \geq\right.$ 0.978).

Table 1. Langmuir Parameters $\left(K_{\mathrm{eq}}\right.$ and $\left.v_{\max }\left(=\Delta C_{\max } / C_{0}\right)\right)$ for Sensor Devices Coated with a Matrimid Layer Containing Different Amounts of $\mathrm{NH}_{2}$-MIL-53(Al) as Function of Different Vapor Concentrations of Methanol

$\begin{array}{ccccc}\begin{array}{c}\text { MOF loading } \\ \text { (wt \%) }\end{array} & \begin{array}{c}K_{\mathrm{eq}} \\ \left(\mathrm{bar}^{1}\right)\end{array} & \begin{array}{c}v_{\max } \\ (\%)\end{array} & R^{2} & \begin{array}{c}v_{\max , \mathrm{MOF}} \text { calculated } \\ (\%)\end{array} \\ 0 & 120 & 4.8 & 0.996 & 0 \\ 20 & 132 & 8.9 & 0.999 & 25 \\ 40 & 241 & 12.9 & 0.995 & 25 \\ 50 & 368 & 6.1 & 0.978 & 7.4 \\ 60 & 264 & 7.1 & 0.998 & 8.6 \\ 100^{a} & 860 & 4.4 & 0.947 & 4.4\end{array}$

${ }^{a}$ Sensor devices with 100 wt \% MOF showed deviation from Langmuir behavior at low concentrations (1000-2000 ppm $(0.1-0.2 \%)$ ) of methanol.

The $100 \mathrm{wt} \%$ coating devices showed a slight deviation from Langmuir behavior at low methanol concentrations. This can be attributed to additional structural changes in the $\mathrm{NH}_{2}$-MIL$53(\mathrm{Al})$ due to the initial adsorption of methanol in freely accessible $\mathrm{MOF}{ }^{38}$ which resulted in a significant transition in the response between $1000(0.1 \%)$ and $2000 \mathrm{ppm}(0.2 \%)$ of methanol in devices coated with 100 wt \% MOF (Figure 4b).

Further, the equilibrium affinity constants of experiments using devices with $\mathrm{NH}_{2}$-MIL-53( $\left.\mathrm{Al}\right)(100 \%)$ and a Matrimid coating show that both MOF and polymer have an intrinsic affinity toward methanol. MOF particles showed higher affinity as expected due to the hydrophilic nature of the pores and the high porosity. All composite-coated devices showed the affinity constants in-between these two extreme cases with 40 wt \% showing the highest saturation response with an increase of $12.9 \%$, compared to $4.8 \%$ and $4.4 \%$ for the only Matrimid (0 wt $\%)$ and only-MOF (100 wt \%) coated devices, respectively.

On the basis of these saturation responses and the mass fraction of MOF, the contribution of MOF toward the response $\left(v_{\text {max }, \mathrm{MOF}}\right)$ was calculated in these composite films using eq 3 :

$$
\nu_{\max }=\nu_{\mathrm{max}, \mathrm{MOF}} \times w_{\mathrm{MOF}}+\nu_{\mathrm{max}, \text { Matrimid }} \times w_{\text {Matrimid }}
$$

where $v$ and $w$ are the equilibrium response and the mass fraction of MOF or Matrimid, respectively. It was assumed that the adsorption contributions of both Matrimid and $\mathrm{NH}_{2}$-MIL53(Al) are additive in the total response. The contribution of Matrimid was determined as the mass fraction of the MOF-free Matrimid coated device. Expectedly, the calculated contribution of MOF toward the relative response doubled over increase in loading from $20 \mathrm{wt} \%\left(v_{\mathrm{max}, \mathrm{MOF}} \times w_{\mathrm{MOF}}=5.0 \%\right)$ to $40 \mathrm{wt} \%$ $\left(v_{\text {max }, \mathrm{MOF}} \times w_{\mathrm{MOF}}=10.0 \%\right)$. From both cases, the saturation response $\left(v_{\max , \mathrm{MOE}}\right)$ of the MOF-only $(100 \mathrm{wt} \%)$ device was calculated to be $25 \%$ which is nearly 6 times higher than the observed response $(4.4 \%)$. Such decrease in $v_{\max }$ (also in case of devices with 50 and 60 wt \% MOF (Table 1)) can be associated, as discussed above, to the decreased adhesion in these highly loaded devices.

In summary, from this comparative study it can be concluded that $40 \mathrm{wt} \%$ showed the highest capacitive change and that for increasing MOF loadings there was a transition at $40 \mathrm{wt} \%$ from polymer-driven adsorption to a MOF-driven adsorption.

3.3. Sensitivity and Selectivity. Next to sensor responses and kinetics, another important parameter to consider for a sensor device is its cross-sensitivity toward different analytes. The 40 wt \% MOF-modified sensor devices were exposed to water, methanol, ethanol, and 2-propanol to study its crosssensitivity (Figures 5 and S8). Upon exposure to $20000 \mathrm{ppm}$ (2\%) of each analyte, the equilibrium response decreased and $\tau_{0.5}$ increased with increase of molecular size of the analyte (water, methanol, ethanol, and 2-propanol) (Figure 5a). A 10fold and 70 -fold increase in $\tau_{0.5}$ for ethanol and 2-propanol,
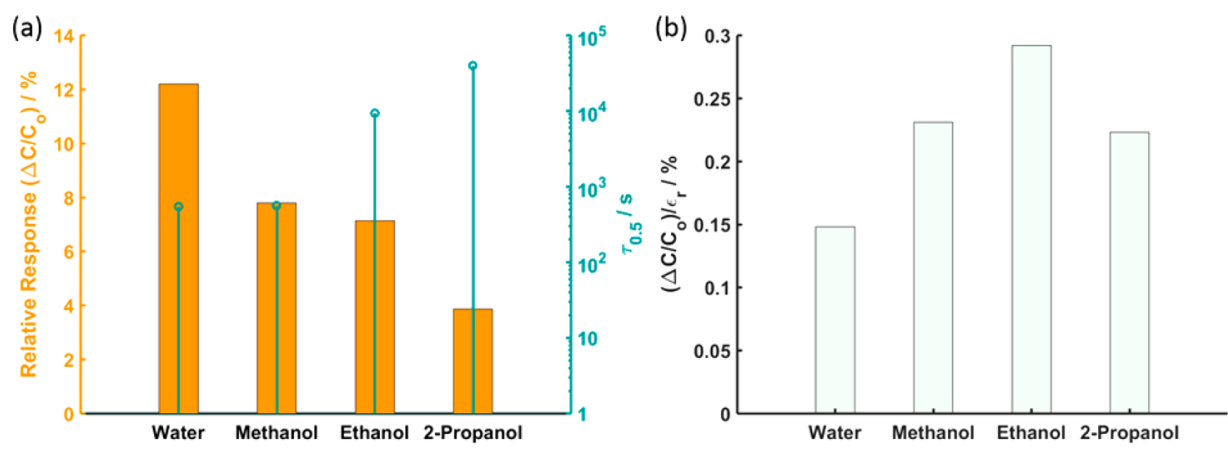

Figure 5. (a) Relative response of a sensor device containing $40 \mathrm{wt} \% \mathrm{MOF}$ in a Matrimid layer upon the exposure to $20000 \mathrm{ppm}$ (2\%) of different analytes (orange) and the corresponding half-time for saturation, $\tau_{0.5}$ (blue). (b) Relative response normalized to the relative dielectric constant. 
respectively, compared to methanol and water clearly indicates slower uptakes, likely due to a slower diffusion through the Matrimid polymer composite. ${ }^{29}$ In order to correlate with the analyte concentration, the equilibrium responses were normalized to the relative dielectric constant $\left(\varepsilon_{\mathrm{r}, \text { Water }}=78.3\right.$, $\varepsilon_{\mathrm{r}, \text { Methanol }}=32.7, \varepsilon_{\mathrm{r}, \text { Ethanol }}=24.5$, and $\varepsilon_{\mathrm{r} \text {,Propanol }}=18$, Figure $5 \mathrm{~b}$ ) Now, it can be seen that the uptake of analytes by the MOFMatrimid composite sensor increases from water to methanol to ethanol. This is well in line with the known higher affinity of Matrimid toward ethanol and methanol than water. ${ }^{46,47}$ Even though the affinity of Matrimid toward 2-propanol was also higher, ${ }^{46,47}$ it still resulted in a decrease in the normalized response. This decrease along with the slower uptake can be attributed to the size-exclusion effect of the Matrimid as previously observed, with no response toward 2-propanol in only Matrimid based sensors. ${ }^{29}$ In contrast, the presence of a higher amount of MOF in the polymer matrix allowed some adsorption of this analyte.

Moreover, with significant responses and similar $\tau_{0.5}$ toward water and methanol, these sensor devices show sensitivity toward both analytes. In order to study this cross-sensitivity further, the behavior of devices with different MOF loadings was compared on exposure to $1000 \mathrm{ppm}$ of water and methanol at $28{ }^{\circ} \mathrm{C}$ (Figure 6a). The change in the response was normalized with respect to the relative dielectric constant of the analyte to make interpretation in terms of change of concentrations in the affinity layer possible.

Figure 6a shows that sensors with only Matrimid as affinity layer exhibit a higher response toward methanol than to water. This is due to the intrinsic higher affinity of Matrimid toward methanol compared to water. ${ }^{29,46}$ For sensors having an affinity layer of pure MOF, the response toward water is slightly larger than toward methanol. This is attributed to the slightly hydrophilic nature of the MOF (certainly the MOF is more hydrophilic than the polymer). For the MOF-Matrimid composite systems, the uptake of water and methanol is always higher than for the pure Matrimid system, with a maximum response (uptake) observed around 40 wt \% MOF.

In Figure $6 \mathrm{~b}$ the sensor response to varying methanol concentrations in the range of 1000 to $8000 \mathrm{ppm}$ in steps of $1000 \mathrm{ppm}$ in the presence of a fixed water concentration of $5000 \mathrm{ppm}$ is presented. The sensor has an affinity layer containing $40 \mathrm{wt} \% \mathrm{MOF}$ in Matrimid. The response is similar (after correction for the fixed contribution of water to the capacitive response) to the response observed in the absence of water (Figure 4a), indicating that there is no competition for water and methanol affinity, and both analytes do behave as if the other component is not present.

In Figure $6 \mathrm{c}$ the sensor response to a fixed concentration of methanol of $5000 \mathrm{ppm}(0.5 \%)$ is shown in the presence of varying water concentrations $(0-10000 \mathrm{ppm}(0-1 \%))$. In the investigated range until $6000 \mathrm{ppm}(0.6 \%)$ of water, it is observed that the capacitance increase is independent of the water concentration, confirming that methanol and water adsorption is noncompetitive. Deviation was observed at still higher water concentration, where condensation in the pores is likely to occur.

3.4. Effect of Temperature on Sensor Response. To get further insight into the sensing process, adsorption studies of methanol and water were also carried out at different temperatures. A sensor device with 40 wt $\% \mathrm{NH}_{2}$-MIL53(Al) in Matrimid and a MOF-free Matrimid (0 wt \%) were exposed to $1000 \mathrm{ppm}$ (0.1\%) of methanol and $1000 \mathrm{ppm}$
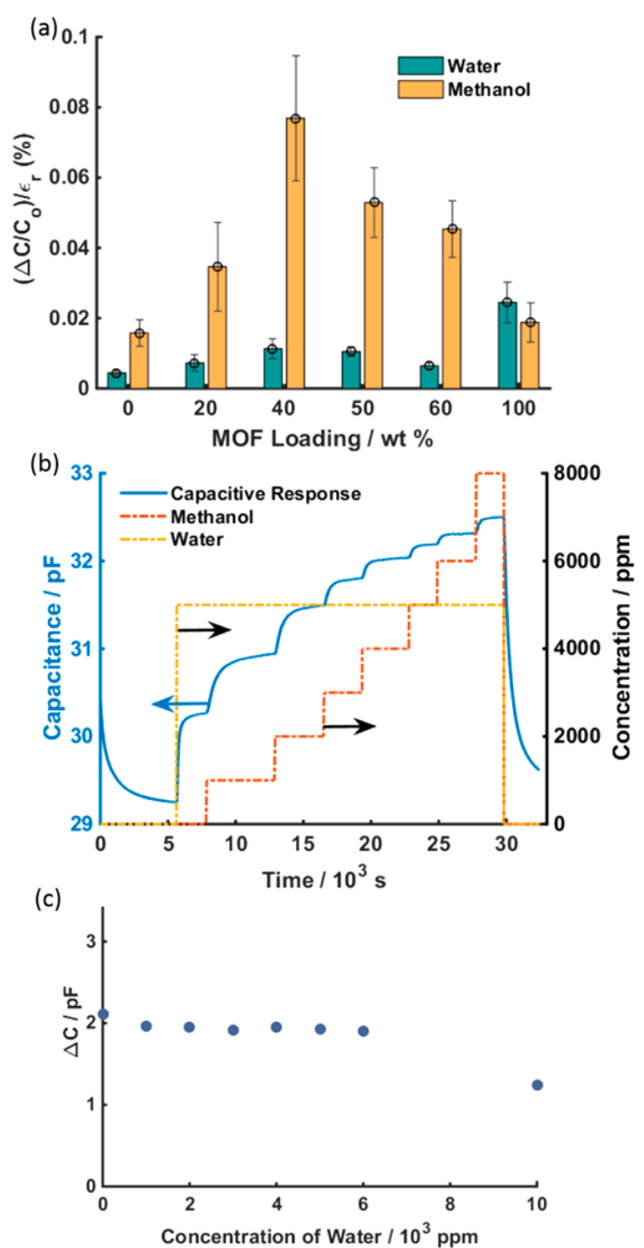

Figure 6. (a) Relative response normalized to relative dielectric constant of sensor devices containing different loadings of $\mathrm{NH}_{2}$-MIL$53(\mathrm{Al})$ in a Matrimid layer upon the exposure to $1000 \mathrm{ppm}(0.1 \%)$ of water and methanol. The error-bars were obtained from the measurements performed on 3 independently replicated devices. (b) The sensor response of a device with $40 \mathrm{wt} \% \mathrm{NH}_{2}-\mathrm{MIL}-53(\mathrm{Al})$ in Matrimid toward different concentrations (1000 to $8000 \mathrm{ppm}$ ( 0.1 to $0.8 \%)$ in steps of $1000 \mathrm{ppm}(0.1 \%)$, indicated by right axis) of methanol in the presence of $5000 \mathrm{ppm}(0.5 \%)$ of water. (c)) The capacitance change of a device with 40 wt $\% \mathrm{NH}_{2}$-MIL-53(Al) in Matrimid toward exposure of $5000 \mathrm{ppm}(0.5 \%)$ of methanol. Each measurement point was obtained at different initial water concentrations as indicated by the $x$-axis. The temperature was kept at $28^{\circ} \mathrm{C}$.

(0.1\%) of water at different temperatures $(301-323 \mathrm{~K})$. The effect of the operating temperature on sensing performance is clearly visible in Figures 7 and S9, showing an Arrhenius plot of the relative response $(v)$ (defined as $\Delta C / C_{0}$ ) versus the absolute temperature $(T)$. At higher temperatures, the capacitance change $\Delta C$ decreases, indicating that less analyte is adsorbed by the affinity layer, as expected.

For the classical Arrhenius behavior applies:

$$
\nu=\nu_{0} \mathrm{e}^{-\frac{\Delta H}{R T}}
$$

where $v_{0}$ and $R$ indicate a pre-exponential constant and the gas constant, respectively. $\Delta H$ represents the difference in activation energy of adsorption and desorption and $v$ represents the relative change in the capacitance $\left(\Delta C / C_{0}\right)$. From the exponential fit of the Arrhenius plot (Figure 7 ), we have deduced the value of $\Delta H$. 

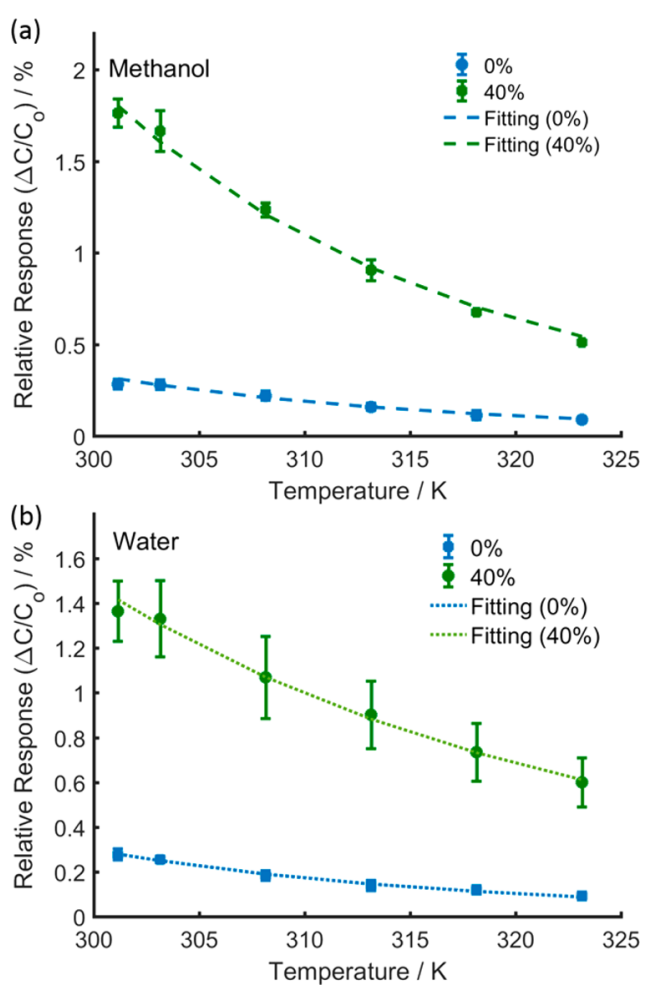

Figure 7. Arrhenius relation between the relative response $(v=\Delta C /$ $C_{0}$ ) and the temperature $(T)$ for devices coated with $40 \mathrm{wt} \%-\mathrm{NH}_{2}-$ MIL-53(Al) in Matrimid (green) and Matrimid only (blue) on the exposure to $1000 \mathrm{ppm}(0.1 \%)$ of methanol (a) and $1000 \mathrm{ppm}(0.1 \%)$ of water (b) at different temperatures. The error-bars were obtained from three measurements done on the same device.

The enthalpy of adsorption $(\Delta H)$ for devices with only Matrimid-coated devices was calculated to be $-44.4 \pm 3.0 \mathrm{~kJ} /$ mol and $-42.6 \pm 9.2 \mathrm{~kJ} / \mathrm{mol}$ for methanol and water, respectively. A negative value of $\Delta H$ confirms the exothermic nature of the process, with similar affinities for methanol and water. For devices with 40 wt \% MOF loading, the enthalpy of adsorption $(\Delta H)$ was found to be $-44.1 \pm 1.3 \mathrm{~kJ} / \mathrm{mol}$ and $-30.9 \pm 1.5 \mathrm{~kJ} / \mathrm{mol}$ for methanol and water, respectively, clearly indicating higher affinity for methanol than for water. Moreover, $\Delta H$ has become less favorable by the addition of MOF to the polymer for adsorption of water. We tentatively suggest that these differences in $\Delta H$ can be related to polymer rigidification near the MOF interface. ${ }^{44,45}$ This rigidification would result in a stronger barrier toward water than toward (the less polar) methanol.

\section{CONCLUSIONS}

In this study, we have investigated affinity layers composed of varying amounts of $\mathrm{NH}_{2}$-MIL-53(Al) MOF mixed in a Matrimid polymer and deposited on top of meander-patterned aluminum electrodes for capacitive sensing in the presence of different analytes in the gas phase. As analytes, we have investigated water, methanol, ethanol, and 2-propanol. The capacitive response toward methanol and water was enhanced by the incorporation of the MOFs in the polymeric matrix. The optimum composition was found at $\sim 40$ wt $\%$ of MOF, showing an up to 5-fold increase in equilibrium response for $1000 \mathrm{ppm}$ of methanol compared with only Matrimid coated devices. The response time also increased with increasing MOF concentration (up-to $40 \mathrm{wt} \%$ ), which is attributed to polymer rigidification near the MOF-polymer interface. Devices containing $\geq 50$ wt $\%$ MOF in Matrimid resulted in brittle and cracked films. The equilibrium response and response time decreased in these devices compared to devices with $40 \mathrm{wt} \%$ MOF and became closer to the equilibrium response and response time shown by a polymer-free, MOF-coated sensor device. The decrease in the response behavior was attributed to the poor adhesion. Quantitative analysis of the sensor devices indicated Langmuir behavior showing a maximum affinity $\left(K_{\mathrm{eq}}\right.$ $=860 \mathrm{bar}^{-1}$ ) for the polymer-free, MOF-coated devices. The affinity of all composite devices was found to be in-between those of $100 \%$ MOF and $100 \%$ Matrimid-coated devices, with the 40 wt \% devices showing a maximum saturation response. Cross-sensitivity studies showed the ability of the devices to kinetically distinguish between alcohols and water. This is based on the molecular size of the analyte resulting in a $\sim 70$ times increased $\tau_{0.5}$ for 2-propanol for the $40 \mathrm{wt} \%$-MOF-polymer sensor compared to methanol and water. Sensing studies with mixtures of water and methanol indicated that both molecules have independent contributions. For water this additive effect to the detection of methanol holds up to concentrations of $6000 \mathrm{ppm}$. The temperature dependency of the sensor devices showed an Arrhenius-type behavior with a stronger methanol adsorption than water adsorption, confirming a higher affinity toward methanol. With differences in response sensitivity and kinetics for devices coated with affinity layers with different MOF-polymer ratios, quantitative fingerprinting of various analytes is within reach by utilizing sensor arrays with different MOF-polymer fractions and types at different operational temperatures.

\section{ASSOCIATED CONTENT}

\section{Supporting Information}

The Supporting Information is available free of charge on the ACS Publications website at DOI: 10.1021/acsami.7b02630.

XRD and TEM data of $\mathrm{NH}_{2}$-MIL-53(Al) nanoparticles (Figures S1 and S2), thickness profiles, SEM data, capacitive sensor response, methanol adsorption isotherms, concentration-dependent sensor response (and Langmuir fittings), cross-sensitivity data, and temperature-dependent sensor response of devices with different composite coatings (Figures S3-S9) (PDF)

\section{AUTHOR INFORMATION}

\section{Corresponding Author}

*E-mail: louis.desmet@wur.nl (L.C.P.M.d.S.).

ORCID $\odot$

Sumit Sachdeva: 0000-0001-5513-4880

Freek Kapteijn: 0000-0003-0575-7953

Ernst J. R. Sudhölter: 0000-0003-3296-953X

Louis C. P. M. de Smet: 0000-0001-7252-4047

Notes

The authors declare no competing financial interest.

\section{ACKNOWLEDGMENTS}

This work was supported by NanoNextNL, a micro and nanotechnology consortium of the Government of The Netherlands and 130 partners. L.C.P.M.d.S. acknowledges the European Research Council (ERC) for a Consolidator Grant, which is part of the European Union's Horizon 2020 research and innovation programme (grant agreement No 682444). We 
also thank Mr. Duco Bosma and Mr. Bart Boshuizen from TU Delft for technical and LABVIEW support.

\section{REFERENCES}

(1) Sun, Y.-F.; Liu, S.-B.; Meng, F.-L.; Liu, J.-Y.; Jin, Z.; Kong, L.-T.; Liu, J.-H. Metal Oxide Nanostructures and Their Gas Sensing Properties: A Review. Sensors 2012, 12, 2610.

(2) Wales, D. J.; Grand, J.; Ting, V. P.; Burke, R. D.; Edler, K. J.; Bowen, C. R.; Mintova, S.; Burrows, A. D. Gas sensing using porous materials for automotive applications. Chem. Soc. Rev. 2015, 44, 42904321.

(3) Liu, X.; Cheng, S.; Liu, H.; Hu, S.; Zhang, D.; Ning, H. A Survey on Gas Sensing Technology. Sensors 2012, 12, 9635.

(4) Jariwala, D.; Sangwan, V. K.; Lauhon, L. J.; Marks, T. J.; Hersam, M. C. Carbon Nanomaterials for Electronics, Optoelectronics, Photovoltaics, and Sensing. Chem. Soc. Rev. 2013, 42, 2824-2860.

(5) Bai, J.; Zhou, B. Titanium Dioxide Nanomaterials for Sensor Applications. Chem. Rev. 2014, 114, 10131-10176.

(6) Bai, H.; Shi, G. Gas Sensors Based on Conducting Polymers. Sensors 2007, 7, 267.

(7) Potyrailo, R. A.; Surman, C.; Nagraj, N.; Burns, A. Materials and Transducers Toward Selective Wireless Gas Sensing. Chem. Rev. 2011, 111, 7315-7354.

(8) Miller, D. R.; Akbar, S. A.; Morris, P. A. Nanoscale metal oxidebased heterojunctions for gas sensing: A review. Sens. Actuators, B 2014, 204, 250-272.

(9) Gosangi, R.; Gutierrez-Osuna, R. Active Temperature Modulation of Metal-Oxide Sensors for Quantitative Analysis of Gas Mixtures. Sens. Actuators, B 2013, 185, 201-210.

(10) Kreno, L. E.; Leong, K.; Farha, O. K.; Allendorf, M.; Van Duyne, R. P.; Hupp, J. T. Metal-Organic Framework Materials as Chemical Sensors. Chem. Rev. 2012, 112, 1105-1125.

(11) Czaja, A. U.; Trukhan, N.; Muller, U. Industrial applications of metal-organic frameworks. Chem. Soc. Rev. 2009, 38, 1284-1293.

(12) Furukawa, H.; Cordova, K. E.; O'Keeffe, M.; Yaghi, O. M. The Chemistry and Applications of Metal-Organic Frameworks. Science 2013, 341, 1230444.

(13) Falcaro, P.; Ricco, R.; Doherty, C. M.; Liang, K.; Hill, A. J.; Styles, M. J. MOF Positioning Technology and Device Fabrication. Chem. Soc. Rev. 2014, 43, 5513-5560.

(14) Mason, J. A.; Veenstra, M.; Long, J. R. Evaluating Metal-Organic Frameworks for Natural Gas Storage. Chemical Science 2014, 5, 32-51.

(15) Stavila, V.; Talin, A. A.; Allendorf, M. D. MOF-based Electronic and Opto-Electronic Devices. Chem. Soc. Rev. 2014, 43, 5994-6010.

(16) $\mathrm{Hu}, \mathrm{Z}$.; Deibert, B. J.; Li, J. Luminescent metal-organic frameworks for chemical sensing and explosive detection. Chem. Soc. Rev. 2014, 43, 5815-5840.

(17) Shekhah, O.; Liu, J.; Fischer, R. A.; Woll, C. MOF Thin Films: Existing and Future Applications. Chem. Soc. Rev. 2011, 40, 10811106.

(18) Sapsanis, C.; Omran, H.; Chernikova, V.; Shekhah, O.; Belmabkhout, Y.; Buttner, U.; Eddaoudi, M.; Salama, K. Insights on Capacitive Interdigitated Electrodes Coated with MOF Thin Films: Humidity and VOCs Sensing as a Case Study. Sensors 2015, 15, 18153.

(19) Campbell, M. G.; Sheberla, D.; Liu, S. F.; Swager, T. M.; Dincă, M. $\mathrm{Cu}_{3}$ (hexaiminotriphenylene) $)_{2}$ : An Electrically Conductive 2D Metal-Organic Framework for Chemiresistive Sensing. Angew. Chem., Int. Ed. 2015, 54, 4349-4352.

(20) Achmann, S.; Hagen, G.; Kita, J.; Malkowsky, I.; Kiener, C.; Moos, R. Metal-Organic Frameworks for Sensing Applications in the Gas Phase. Sensors 2009, 9, 1574.

(21) Seoane, B.; Coronas, J.; Gascon, I.; Benavides, M. E.; Karvan, O.; Caro, J.; Kapteijn, F.; Gascon, J. Metal-Organic Framework Based Mixed Matrix Membranes: a Solution for Highly Efficient CO2 Capture? Chem. Soc. Rev. 2015, 44, 2421-2454.

(22) Sabetghadam, A.; Seoane, B.; Keskin, D.; Duim, N.; Rodenas, T.; Shahid, S.; Sorribas, S.; Guillouzer, C. L.; Clet, G.; Tellez, C.; Daturi, M.; Coronas, J.; Kapteijn, F.; Gascon, J. Metal Organic Framework Crystals in Mixed-Matrix Membranes: Impact of the Filler
Morphology on the Gas Separation Performance. Adv. Funct. Mater. 2016, 26, 3154-3163.

(23) Shahid, S.; Nijmeijer, K.; Nehache, S.; Vankelecom, I.; Deratani, A.; Quemener, D. MOF-Mixed Matrix Membranes: Precise Dispersion of MOF Particles with Better Compatibility via a Particle Fusion Approach for Enhanced Gas Separation Properties. J. Membr. Sci. 2015, 492, 21-31.

(24) Salavagione, H. J.; Diez-Pascual, A. M.; Lazaro, E.; Vera, S.; Gomez-Fatou, M. A. Chemical Sensors Based on Polymer Composites with Carbon Nanotubes and Graphene: the Role of the Polymer. $J$. Mater. Chem. A 2014, 2, 14289-14328.

(25) Hatchett, D. W.; Josowicz, M. Composites of Intrinsically Conducting Polymers as Sensing Nanomaterials. Chem. Rev. 2008, 108, 746-769.

(26) Wang, S.; Kang, Y.; Wang, L.; Zhang, H.; Wang, Y.; Wang, Y. Organic/Inorganic Hybrid Sensors: A Review. Sens. Actuators, B 2013, 182, 467-481.

(27) Harsányi, G. Polymer Films in Sensor Applications: a Review of Present Uses and Future Possibilities. Sens. Rev. 2000, 20, 98-105.

(28) Lau, W. J.; Ismail, A. F.; Misdan, N.; Kassim, M. A. A Recent Progress in Thin Film Composite Membrane: A Review. Desalination 2012, 287, 190-199.

(29) Sachdeva, S.; Soccol, D.; Gravesteijn, D. J.; Kapteijn, F.; Sudhölter, E. J. R.; Gascon, J.; de Smet, L. C. P. M. Polymer-Metal Organic Framework Composite Films as Affinity Layer for Capacitive Sensor Devices. ACS Sens. 2016, 1, 1188-1192.

(30) Scholes, C. A.; Tao, W. X.; Stevens, G. W.; Kentish, S. E. Sorption of Methane, Nitrogen, Carbon Dioxide, and Water in Matrimid 5218. J. Appl. Polym. Sci. 2010, 117, 2284-2289.

(31) Rodenas, T.; van Dalen, M.; Serra-Crespo, P.; Kapteijn, F.; Gascon, J. Mixed Matrix Membranes Based on $\mathrm{NH}_{2}$-Functionalized MIL-type MOFs: Influence of Structural and Operational Parameters on the $\mathrm{CO}_{2} / \mathrm{CH}_{4}$ Separation Performance. Microporous Mesoporous Mater. 2014, 192, 35-42.

(32) Couck, S.; Denayer, J. F. M.; Baron, G. V.; Rémy, T.; Gascon, J.; Kapteijn, F. An Amine-Functionalized MIL-53 Metal-Organic Framework with Large Separation Power for $\mathrm{CO} 2$ and CH4. J. Am. Chem. Soc. 2009, 131, 6326-6327.

(33) Zornoza, B.; Tellez, C.; Coronas, J.; Gascon, J.; Kapteijn, F. Metal Organic Framework based Mixed Matrix Membranes: An Increasingly Important Field of Research with a Large Application Potential. Microporous Mesoporous Mater. 2013, 166, 67-78.

(34) Dang, Z.-M.; Nan, C.-W.; Xie, D.; Zhang, Y.-H.; Tjong, S. C. Dielectric Behavior and Dependence of Percolation Threshold on the Conductivity of Fillers in Polymer-Semiconductor Composites. Appl. Phys. Lett. 2004, 85, 97-99.

(35) Igreja, R.; Dias, C. J. Analytical evaluation of the interdigital electrodes capacitance for a multi-layered structure. Sens. Actuators, A 2004, 112, 291-301.

(36) Igreja, R.; Dias, C. J. Dielectric response of interdigital chemocapacitors: The role of the sensitive layer thickness. Sens. Actuators, B 2006, 115, 69-78.

(37) Van Gerwen, P.; Laureyn, W.; Laureys, W.; Huyberechts, G.; Op De Beeck, M.; Baert, K.; Suls, J.; Sansen, W.; Jacobs, P.; Hermans, L.; Mertens, R. Nanoscaled Interdigitated Electrode Arrays for Biochemical Sensors. Sens. Actuators, B 1998, 49, 73-80.

(38) Serra-Crespo, P.; Gobechiya, E.; Ramos-Fernandez, E. V.; JuanAlcañiz, J.; Martinez-Joaristi, A.; Stavitski, E.; Kirschhock, C. E. A.; Martens, J. A.; Kapteijn, F.; Gascon, J. Interplay of Metal Node and Amine Functionality in $\mathrm{NH}_{2}$-MIL-53: Modulating Breathing Behavior through Intra-Framework Interactions. Langmuir 2012, 28, 1291612922.

(39) Yaws, C. L. The Yaws Handbook of Vapor Pressure: Antoine Coefficients; Gulf Professional Publishing: Houston, TX, 2015.

(40) Thomson, G. W. The Antoine Equation for Vapor-pressure Data. Chem. Rev. 1946, 38, 1-39.

(41) Banerjee, N.; Roy, S.; Sarkar, C. K.; Bhattacharyya, P. High Dynamic Range Methanol Sensor Based on Aligned ZnO Nanorods. IEEE Sens. J. 2013, 13, 1669-1676. 
(42) Arshak, K.; Gaidan, I. Development of a novel gas sensor based on oxide thick films. Mater. Sci. Eng., B 2005, 118, 44-49.

(43) Minelli, M.; Cocchi, G.; Ansaloni, L.; Baschetti, M. G.; De Angelis, M. G.; Doghieri, F. Vapor and Liquid Sorption in Matrimid Polyimide: Experimental Characterization and Modeling. Ind. Eng. Chem. Res. 2013, 52, 8936-8945.

(44) Moore, T. T.; Koros, W. J. Non-Ideal Effects in OrganicInorganic Materials for Gas Separation Membranes. J. Mol. Struct. 2005, 739, 87-98.

(45) Burmann, P.; Zornoza, B.; Téllez, C.; Coronas, J. Mixed Matrix Membranes Comprising MOFs and Porous Silicate Fillers Prepared via Spin Coating for Gas Separation. Chem. Eng. Sci. 2014, 107, 66-75.

(46) Barton, A. F. M. Solubility Parameters. Chem. Rev. 1975, 75, 731-753.

(47) Jiang, L. Y.; Chen, H.; Jean, Y.-C.; Chung, T.-S. Ultrathin Polymeric Interpenetration Network with Separation Performance Approaching Ceramic Membranes for Biofuel. AIChE J. 2009, 55, 7586. 